IOS Press

\title{
Modified Newton-Raphson method to tune feedback gains of control system for standing by functional neuromuscular stimulation following spinal cord injury
}

\author{
Raviraj Nataraj ${ }^{\mathrm{a}, \mathrm{b}, \mathrm{c}, *}$, Musa L. Audu ${ }^{\mathrm{a}, \mathrm{b}, \mathrm{c}}$ and Ronald J. Triolo ${ }^{\mathrm{a}, \mathrm{b}, \mathrm{c}}$ \\ ${ }^{a}$ Louis Stokes Veterans Affairs Medical Center, Motion Study Laboratory, Cleveland, OH, USA \\ ${ }^{\mathrm{b}}$ Department of Biomedical Engineering, Case Western Reserve University, Cleveland, OH, USA \\ ${ }^{\mathrm{c}}$ Department of Orthopaedics, Case Western Reserve University, Cleveland, OH, USA
}

\begin{abstract}
.
BACKGROUND: Functional neuromuscular stimulation (FNS) can restore standing capabilities following spinal cord injury. Feedback control of these systems can optimize performance by reducing the required upper extremity support. However, tuning these control systems can be intensive and clinically inconvenient.

OBJECTIVE: This case study investigated a clinical method to efficiently tune feedback gains for a control system utilizing feedback of total body center of mass acceleration to modulate stimulation levels to targeted paralyzed musculature of the lower extremities and trunk.

METHODS: Gains for this control system were tuned to minimize the stabilization loading by one arm against internal postural perturbations volitionally-generated during manipulation of an object using the other arm. An algorithm based on a modified form of the Newton-Raphson method was employed to find the optimal feedback gains with lower subject effort than that to determine the original tuning curves.

RESULTS: This method accurately ( $<6.2 \%$ error) approximated the optimal gains with $70 \%$ fewer manipulations by the subject. CONCLUSIONS: These results suggest that optimal feedback gains for the specific FNS control system can be determined systematically with considerably less effort than heuristic gain tuning. This demonstrates the potential for devising simple, convenient methods for effective system re-tuning during clinical usage.
\end{abstract}

Keywords: Standing, functional neuromuscular stimulation, spinal cord injury, feedback control system, adaptive algorithm

\section{Introduction}

Functional neuromuscular stimulation (FNS) is a clinically proven intervention to restore basic standing

\footnotetext{
*Corresponding author: Raviraj Nataraj, Ph.D., Louis Stokes Veterans Affairs Medical Center, Motion Study Laboratory, Cleveland, OH 44106, USA. Tel.: +1 440804 4966; E-mail: raviraj. nataraj@gmail.com.
}

capabilities for individuals with complete spinal cord injury (SCI) [4]. Current clinical implementation of FNS standing systems involves application of continuous, open-loop stimulation to extensor musculature at the knees, hips, and trunk. Users need to stabilize against postural perturbations by applying loads on an assistive device (e.g., walker) with their arms. This accelerates upper-body fatigue and compromises the ability to perform functional standing tasks such as 
reaching and manipulating objects. Previous studies have examined feedback control to modulate stimulation and produce automatic postural corrections against disturbances $[2,6,7]$. However, these investigations restricted control to single joints and constrained planes of movement. Approaches involving simultaneous control of multiple joints and unconstrained, three-dimensional (3-D) movements would hasten clinical acceptance of closed-loop FNS standing. To this end, our group has developed control systems that modulate stimulation to muscles spanning the ankles, knees, hips, and trunk for comprehensive standing balance. We have demonstrated that feedback control can notably reduce the upper extremity (UE) loading required to stabilize against postural perturbations up to $50 \%$ in computer simulation [8-10] and 27-33\% under live-subject conditions [12, 13] compared to constant muscle excitation levels. While the procedures implemented to heuristically tune the feedback gains for these controllers were appropriate for initial evaluations, their repeated implementation would be clinically inconvenient by requiring substantial subject time and effort for periodic re-tuning over long-term usage. This paper demonstrates that a more efficient clinical method to determine the optimal feedback gains of FNS control systems for standing utilizing 3-D accelerations of the total body center of mass (COM) as feedback is feasible given presumably concave profiles of the gain tuning curves.

\section{Methods}

\subsection{Subject with SCI and FNS control system}

The subject was a 54-year old female with complete thoracic-level (T4) spinal cord injury. She utilized an FNS standing system that included an implantable stimulator-telemeter [3] and controller employing COM acceleration feedback $[12,13]$. While standing, the subject accessed a support device with instrumented handles to actively track the UE loading applied to resist postural disturbances (Fig. 1). The FNS system incorporated channels of either intramuscular or surface stimulation applied bilaterally to the: erector spinae*, gluteus maximus, gluteus medius*, rectus femoris*, semimembranosus, thigh adductors*, tibialis anterior*, triceps surae*, and vasti. The channels denoted by '*' were under feedback control, while the remaining outputted constant stimulation for stand-

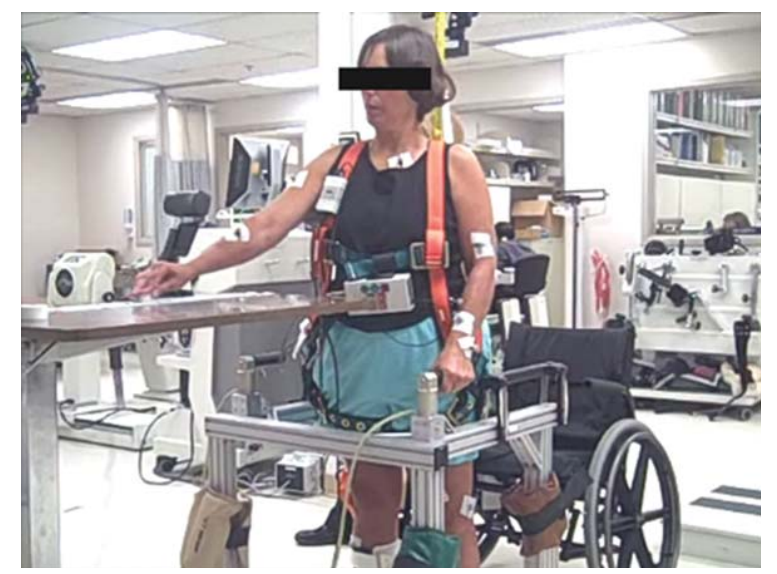

Fig. 1. Individual with complete, thoracic (T4) level spinal cord injury standing with stimulation to musculature spanning trunk and lower extremities. She stabilized with her left hand while performing manual slide-shifts of an object with her right hand.

ing maintenance. Measurements from body-mounted 3-D accelerometers were input to a linear regression model to estimate feedback COM acceleration in the anterior-posterior (AP) and medial-lateral (ML) dimensions relative to a globally-fixed anatomical reference frame. Each COM acceleration feedback value was multiplied by a respective constant gain before driving an artificial neural network (ANN) to produce changes in muscle stimulation to counter those acceleration effects. The relationship between gain value, feedback-input, and stimulation-output is summarized in the following controller relationship:

$$
\bar{S}(t)=H\left(K_{A P} * a c c_{A P}(t), K_{M L} * a c c_{M L}(t)\right)
$$

where ' $\bar{S}(t)$ ' is a vector containing the stimulation levels of all targeted muscles at each time instance ' $t$ ', ' $K$ ' is the gain value multiplying the respective acceleration value ' $a c c$ ' used as feedback-input at ' $t$ ', and ' $H$ ' represents the input-output transformation by an artificial neural network. Similar to classical proportional control, the target feedback-inputs were multiplied by respective gain values prior to transformation to the actuation output variable. In this case, the transformation was non-linear and black-box via the artificial neural network trained to relate changes in induced acceleration of the total body center of mass to optimal stimulation levels as described in [13]. The control system essentially acted as a closed-loop, error-driven controller whereby total body center of mass acceleration was the feedback variable and control actions continued until feedback values became zero. 

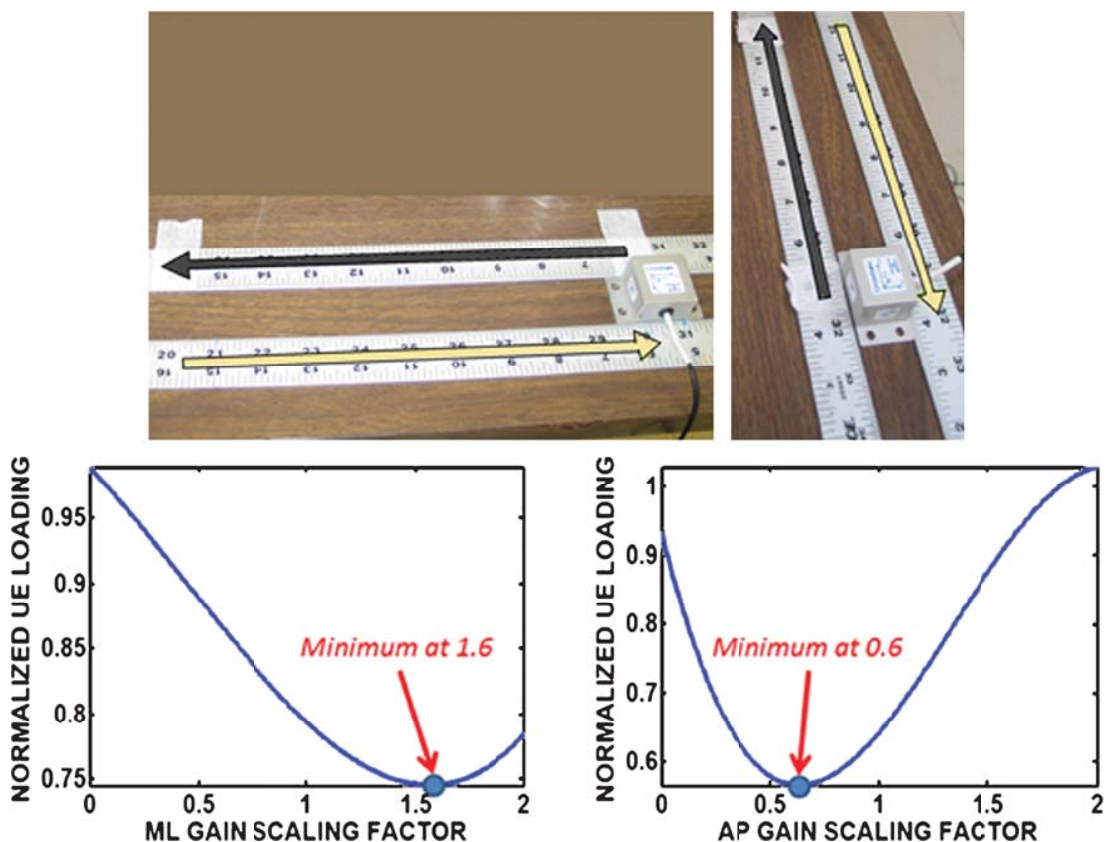

Fig. 2. TOP: Subject manually slide-shifts (dark/light arrow is initial away/return direction) an accelerometer along either the medial-lateral (ML, pictured left) or anterior-posterior (AP, pictured right) dimension. BOTTOM: Third-order polynomial fits for normalized upper extremity (UE) loading versus gain from $[12,13]$.

\subsection{Gain tuning curves from prior investigations}

Investigations to determine the optimal feedback gains that minimize the UE loading this subject applies with her left hand to stabilize against postural perturbations were previously conducted and detailed in [12]. Salient content is summarized in this section for appropriate context. First, optimal feedback gains to resist externally-applied, systematic postural perturbations enacted by linear actuators were determined [13]. Second, these gains were adjusted (pre-multiplied) by optimal gain scaling factors for operation during internal (i.e., generated volitionally by the subject herself) perturbations from right-side reaching and slide-manipulation of a light-weight object as described in [12]. In searching for scaling factors to multiply previously determined gain values, it was presumed that the optimal gains identified for the same subject to resist external perturbations would serve as ideal initial conditions to achieve fast convergence upon optimal scaling factors unique to the task of slide-shifting. Ultimately, each scaling factor $(S F)$ produced a new gain value $(K)$ to multiply respective feedback-input prior to ANN transformation as follows: $K_{\text {new }}=S F * K_{\text {previous }}$. The slide-shifts served as well-controlled surrogates to functional activities of daily living to initially test controller performance. Each slide-shift consisted of moving a light-weight object (accelerometer) between ruler guide-rails from a starting position, to a target-away, and immediately back to the starting position in either the AP or ML dimension (Fig. 2, TOP). The subject performed 20 shifts at a particular gain scaling factor $(0.0,0.25,0.5$, $0.75,1.0,1.5$, and 2.0 ) for each dimension while leftside UE loading was continuously monitored. Offline, the mean UE loading at each scaling factor was calculated and normalized such that the maximum mean UE loading value equaled 1. A 3rd-order polynomial was fit to these mean values for each dimension, and the minimum of each polynomial tuning curve served as the optimal gain scaling factor (0.6 for AP, 1.6 for ML, see Fig. 2, BOTTOM).

\subsection{Modified Newton-Raphson algorithm to find optimal feedback gains}

Since the tuning curves from [12] exhibited simple concave basin profiles, it was hypothesized in this study that an adaptive algorithm with simple iterative root finder could be employed clinically to effectively 
determine the optimal gain values with considerably less subject effort. A modified form of the NewtonRaphson method [5] was developed to converge upon the minimum of each presumed tuning curve. For calculating the next iteration gain scaling factor, the following equation was executed:

$$
x_{k+1}=x_{k}-C^{k} f^{\prime}\left(x_{k}\right)
$$

$k=$ index for current iteration

$x_{k}=$ gain scaling factor for current iteration

$x_{k+1}=$ gain scaling factor for next iteration

$f^{\prime}\left(x_{k}\right)=$ derivative of mean UE loading at $x_{k}$ $C=0.90$, step contraction factor to hasten convergence.

This formulation is modified from classic NewtonRaphson given inclusion of the contraction factor and omission of the second derivative (i.e., $f^{\prime \prime}\left(x_{k}\right)$ ). The contraction factor was employed to hasten convergence, and the second derivative was not calculated due to computational expense (i.e, requiring more trial data) and heuristic observation that steps proportional to $f^{\prime}\left(x_{k}\right)$ (i.e, $f^{\prime \prime}\left(x_{k}\right)=1$ ) consistently yielded acceptable convergence.

Each trial-run of the algorithm began with the gain scaling factor set equal to 1 for the first test iteration. To begin the iteration, the subject performed three consecutive slide-shifts. During the shift periods, defined as intervals over which the total estimated COM acceleration exceeded $100 \mathrm{~mm} / \mathrm{sec}^{2}$ for at least 100 consecutive msec plus an additional $500 \mathrm{msec}$, the changes in leftside UE loading from the beginning of the period were tracked. The subject continued to perform shifts until the standard deviation for mean UE loading across the shift periods was below 1 Newton $(\mathrm{N})$ or a maximum 5 shifts performed. The mean UE loading at the gain scaling factor ' $x$ ' for iteration ' $k$ ' was recorded as $U E_{k}$. To complete the iteration, this process was repeated at gain value 0.1 greater than $x_{k}$ with the UE loading recorded as $U E_{k}^{+}$to compute the function derivative value in (1) with:

$$
f^{\prime}\left(x_{k}\right)=\frac{U E_{k}^{+}-U E_{k}}{0.1}
$$

This iterative process continued until satisfaction of the convergence criterion, whereby the change in the cumulative moving average of $x_{k}$ was $<0.01$. A total of three trial-runs were performed in each dimension for this subject.

\section{Results}

Sample UE loading and corresponding COM acceleration data during five AP slide-shifts are shown in Fig. 3. Dynamic loading applied by the left arm to stabilize during the shifts ranged from 0 to $25 \mathrm{~N}$ and the average loading was $9.9 \mathrm{~N}$. The mean change in UE loading across shift periods was $3.77 \pm 0.73 \mathrm{~N}$. The mean total body COM acceleration during a trial-run was $160 \pm 106 \mathrm{~mm} / \mathrm{sec}^{2}$. Each shift clearly produced a distinct burst of changes in COM acceleration from which muscle stimulation levels were modulated.

The results of three trial-runs in each dimension (AP, ML) are seen in Fig. 4. In all trial-runs, convergence occurred in either 5 or 6 iterations. Using the initial value of 1 , every trial-run adjusted the gain scaling factor towards the expected optimal values of 0.6 and 1.6 in the AP and ML dimensions, respectively, as previously determined [12]. The gain scaling factor converged (final value) between $0.59 \pm 0.06$ and $1.40 \pm 0.13$ in the AP and ML dimensions, respectively. These final values were consistent with the respective optimal scaling factors from [12] with average differential of only $6.2 \%$ across all 6 trialruns. Furthermore, mapping these gain differentials to changes in UE loading according to the respective original tuning curves corresponded to a change of $<2 \%$ in UE loading.
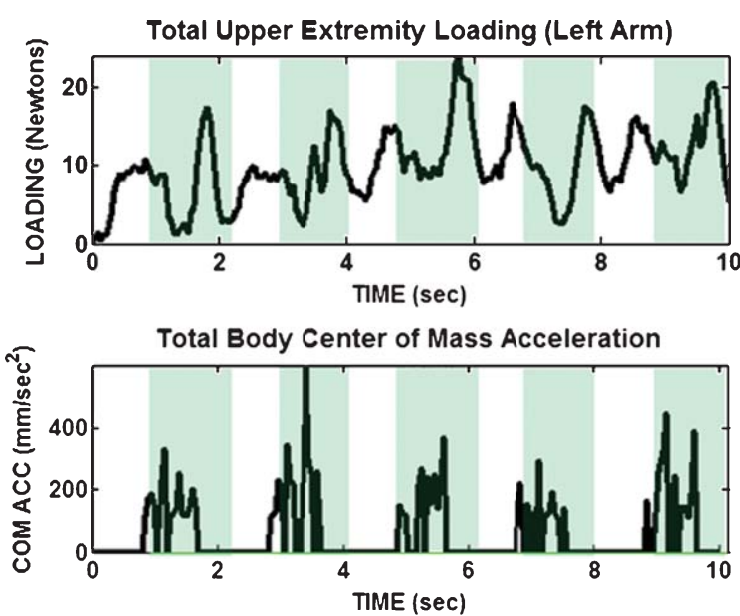

Fig. 3. TOP: Changes in left-side upper extremity (UE) loading to stabilize during five consecutive slide-shifts in anterior-posterior direction. BOTTOM: Corresponding estimate of center of mass acceleration and tracking period (light trace). Note: Accelerations $<100 \mathrm{~mm} / \mathrm{sec}^{2}$ displayed as zero. Shift periods denoted by shaded areas. 
AP Trial-Run\# 1 Final Value $=\mathbf{0 . 6 6}$

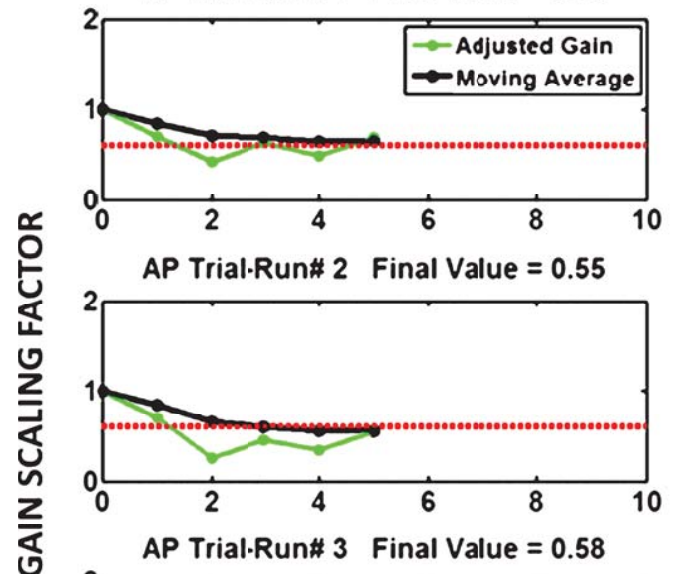

$M L$ Trial-Run\# 1 Final Value $=1.36$

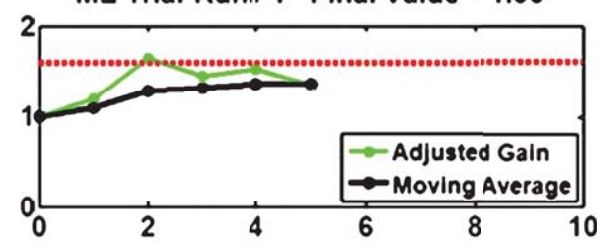

ML Trial-Run\# 2 Final Value $=\mathbf{1 . 5 5}$

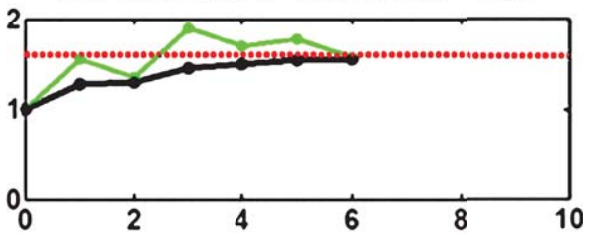

ML Trial-Run\# 3 Final Value $=1.30$

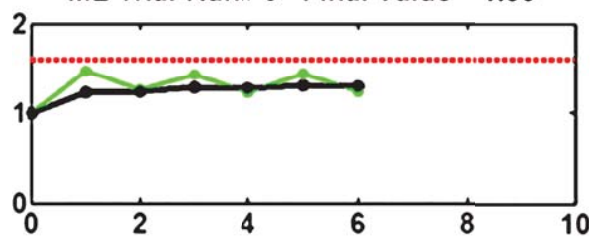

\# ITERATIONS

Fig. 4. Results for three trial-runs applying test algorithm to adapt gain scaling factor towards presumed optimal values (denoted by dotted line) in anterior-posterior (AP) and medial-lateral (ML) dimensions.

Each trial-run was completed in less than 5 minutes. The average total number of shifts the subject performed across all trial runs was $41.5 \pm 4.9$. Compared to the 140 shifts performed in [12] to determine each optimal gain scaling factor, the average number of shifts observed in this study represented a significant $(p<0.001)$ reduction in subject effort of $70.4 \%$.

\section{Discussion}

The results of this pilot study suggest that optimal feedback gains can be efficiently and accurately approximated with considerably less effort than heuristic exploration across an entire range of gain values as typically done with clinical tuning. Convergence towards the optimal values expected was consistently observed in both raw and smoothed scaling factors. These observations demonstrates the basic efficacy of the presented approach and validates the presumption of smooth, basin characteristics of the tuning curves (Fig. 2) previously identified in [12]. Demonstrating basin characteristics of the tuning curves is of primary importance for future developments of simple iterative schemes for effective gain adaptation.
This scheme for adjusting gain factors had certain parameters that were selected ad hoc (e.g., step contraction factor, initial value of gain scaling factor, number of shifts to observe UE loading standard deviation, etc ... ), but these values could be specified more systematically in the future. However, these pilot results still clearly demonstrate that a simple scheme can consistently adjust the gains toward optimal performance. Gains were modified in each direction independently to shape the distinct fore-aft and medial-lateral synergies observed for FNS standing [11]. The raw scaling factors displayed some variability and oscillatory behavior, necessitating use of a moving average to determine convergence (Fig. 4). Considering these values were driven by live human performance, this type of variability is expected and the application of a smoothing tool to isolate a global trend is reasonable. While the optimal feedback gain values presented in the current and previous studies have been based on the performance of the same participant to consistently demonstrate methodology, the need to develop fast and efficient methods in customizing subject-specific controller structures is further highlighted.

Previous studies have outlined the importance of adapting system parameters according to time-varying 
muscle output [1], further highlighting the value of a method that allows controllers to be re-tuned periodically with minimal effort. This study represents a promising basis from which to develop more sophisticated techniques for gain adaptation. Other algorithms (e.g., Levenberg-Marquardt, quasi-Newton) may also be applicable to quickly compute optimal scaling factors. The basic testing paradigm presented in this study is a critical first step in establishing that the system would reliably converge. Furthermore, it is conceivable that a simple device that mimics the laboratory test apparatus could be constructed for periodic system re-tuning during long-term usage at home.

\section{Acknowledgments}

This work has been supported by NIH R01NS040547, NIH T32AR00750525, VA IRB \#2008-027, MHMC IRB \#05-00430, VA AWARD \#A9259-L, and NCATS UL1TR000439. The authors would further like to thank the study participant and the people and facilities of the Cleveland APT and FES Centers of Excellence.

\section{Conflicts of interest}

This work has not previously been published in any other scientific journal or conference. There exists no commercial relationship resulting from this work that may lead to any conflicts of interest. This document has been read and agreed upon by all included authors.

\section{References}

[1] J.J. Abbas and R.J. Triolo, Experimental evaluation of an adaptive feedforward controller for use in functional neuromuscular stimulation systems, IEEE Trans Rehabil Eng $\mathbf{5}$ (1997), 12-22.
[2] J.J. Abbas and H.J. Chizeck, Feedback control of coronal plane hip angle in paraplegic subjects using functional neuromuscular stimulation, IEEE Trans Biomed Eng 38 (1991), 687-698.

[3] N. Bhadra, K.L. Kilgore and P.H. Peckham, Implanted stimulators for restoration of function in spinal cord injury, Med Eng Phys 23 (2001), 19-28.

[4] J.A. Davis, R.J. Triolo, J. Uhlir, C. Bieri, L. Rohde, D Lissy and S. Kukke, Preliminary performance of a surgically implanted neuroprosthesis for standing and transfers-where do we stand? J Rehabil Res Dev 38 (2001), 609-617.

[5] J.E. Dennis Jr. and R.B. Schnabel, Numerical Methods for Unconstrained Optimization and Nonlinear Equations, Prentice Hall, Inc, Englewood Cliffs, NJ, 1983, pp. 86-152.

[6] K.J. Hunt, H. Gollee and R.P. Jaime, Control of paraplegic ankle joint stiffness using FES while standing, Med Eng Phys 38 (2001), 541-555.

[7] M. Moynahan and H.J. Chizeck, Characterization of paraplegic disturbance response during FNS standing, IEEE Trans Rehabil Eng 1 (1993), 43-48.

[8] R. Nataraj, M.L. Audu, R.F. Kirsch and R.J. Triolo, Comprehensive joint-feedback control for standing by functional neuromuscular stimulation following spinal cord injury - a simulation study, IEEE Trans Neural Syst Rehabil Eng $\mathbf{1 8}$ (2010), 646-657.

[9] R. Nataraj, M.L. Audu, R.F. Kirsch and R.J. Triolo, Trunk acceleration for neuroprosthetic control of standing: A pilot study, J App Biomech 28 (2012), 85-92.

[10] R. Nataraj, M.L. Audu, R.F. Kirsch and R.J. Triolo, Center of mass acceleration feedback control for standing by functional neuromuscular stimulation - a simulation study, J Rehabil Res Dev 49 (2012), 279-296.

[11] R. Nataraj, M.L. Audu and R.J. Triolo, Comparing joint kinematics and center of mass accelerations as feedback for control of standing balance by functional neuromuscular stimulation, J NeuroEng Rehab 9 (2012), 1-23.

[12] R. Nataraj, M.L. Audu and R.J. Triolo, Center of mass acceleration feedback control of functional neuromuscular stimulation for standing in presence of internal postural perturbations, J Rehabil Res Dev 49 (2012), 889-912.

[13] R. Nataraj, M.L. Audu and R.J. Triolo, Center of mass acceleration feedback control of functional neuromuscular stimulation for standing against external postural perturbations, IEEE Trans Biomed Eng 60(1) (2013), 10-19. 

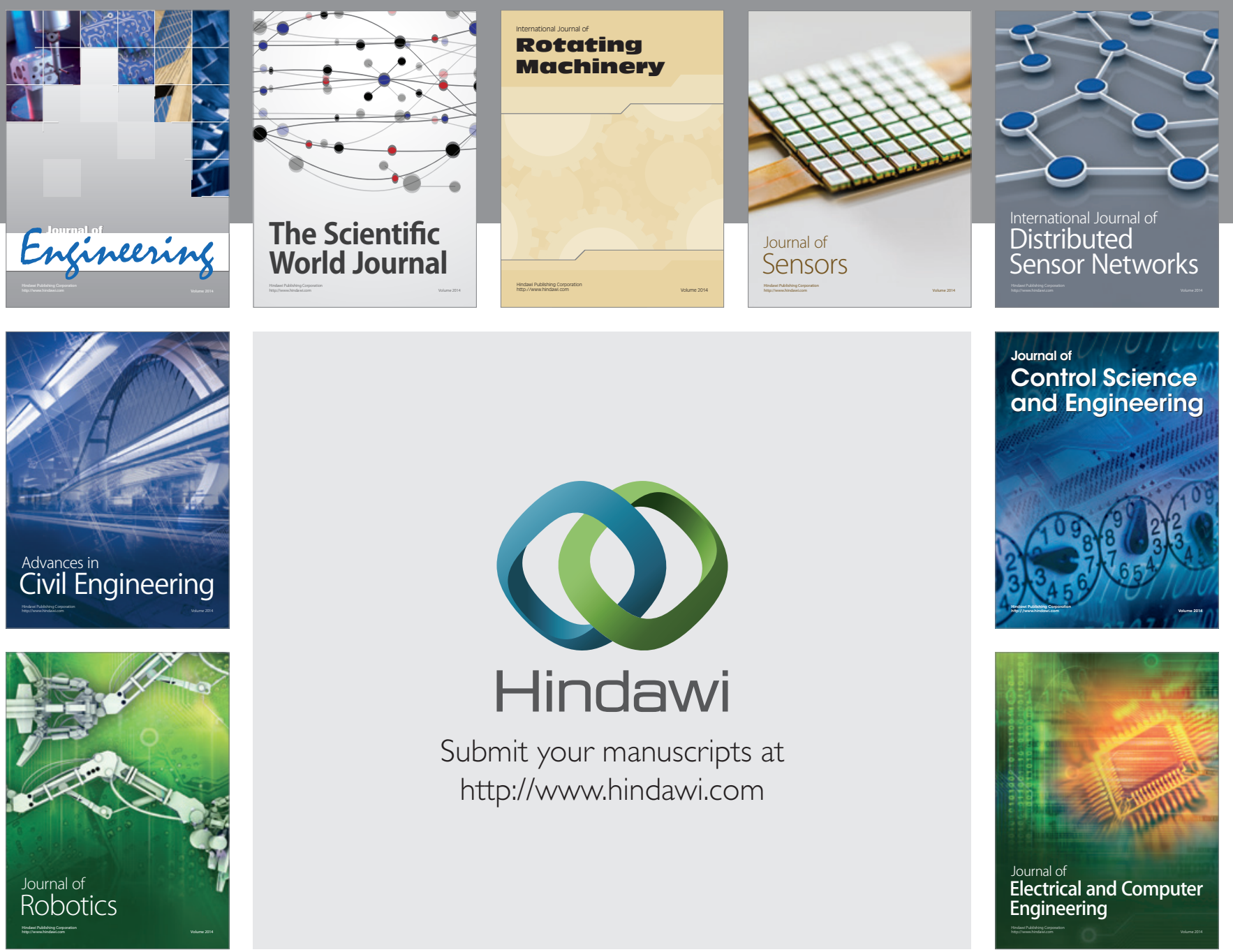

Submit your manuscripts at

http://www.hindawi.com
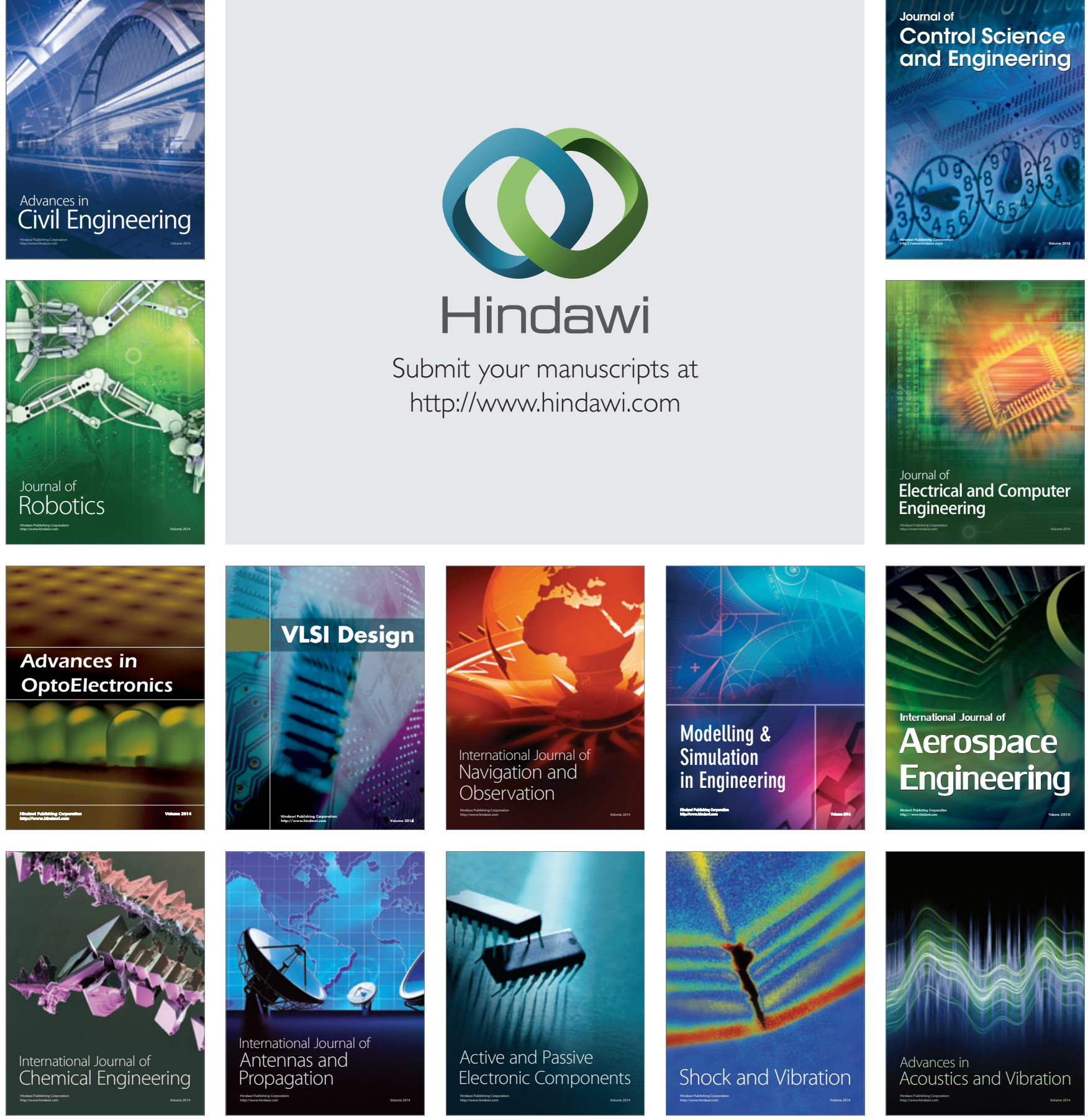